\title{
Cognitive and balance dysfunctions due to the use of zolpidem in the elderly: a systematic review
}

\author{
Guilherme Tavares ${ }^{1 \oplus}$, Gizela Kelmann ${ }^{1 \oplus}$, Francisco Tustumi1,2, ${ }^{1}$, Catherine Nardini Tundisi ${ }^{1 \oplus}$, Bárbara Regina \\ Bruço Silveira $^{1 \oplus}$, Bruno Maximiliano Augusto Colombo Barbosa ${ }^{1 \oplus}$, Diana Bragança Winther ${ }^{1 \odot}$, \\ Eduarda Conte Boutros ${ }^{\oplus}{ }^{\oplus}$, Gabriel dos Santos Villar ${ }^{1}$, Giovanna Brunocilla ${ }^{\oplus}$, Gustavo Rodrigues \\ Caldas Lourenção ${ }^{1 \oplus}$, Jiulia Giovanna Aranha Ferreira ${ }^{1 \oplus}$, Wanderley Marques Bernardo $0^{1,2}$
}

\begin{abstract}
Zolpidem is one of the most widely prescribed hypnotic (non-benzodiazepine) agents for sleep disorder. Recently, an increase in the demand for this drug has been observed, mainly in the elderly population. Objective: This study aims to analyze the acute effect of zolpidem on cognitive and balance dysfunctions in the elderly population. Methods: A study was conducted by two independent researchers in four virtual scientific information bases and included randomized controlled trials. The studies evaluated elderly patients using zolpidem. Cognitive and balance dysfunctions were analyzed. Results: Six articles were included. The mean age of the participants in the studies was 69 years. The following zolpidem dosages were evaluated: 5, 6.25, 10, and $12.5 \mathrm{mg}$. Comparing zolpidem and placebo, relating to the cognitive dysfunctions, there is no statistically significant difference between the groups. However, in relation to balance dysfunctions, there is a statistically significant difference between the intervention and the comparison, favoring placebo. Conclusions: Zolpidem, even in usual doses ( $5 \mathrm{mg}$ and $10 \mathrm{mg}$ ), has shown to increase the risk for balance dysfunctions. However, this does not occur in relation to cognitive changes.
\end{abstract}

Keywords: zolpidem, aged, postural balance, cognitive dysfunction, systematic review.

\section{ALTERAÇõES COGNITIVAS E DE EQUILÍBRIO DEVIDO AO USO DE ZOLPIDEM EM IDOSOS: UMA REVISÃO SISTEMÁTICA}

RESUMO. Zolpidem é um dos agentes hipnóticos (não benzodiazepínicos) mais prescritos para o manejo dos distúrbios do sono. Recentemente, observou-se um aumento na demanda por esse medicamento, principalmente pela população idosa. Objetivo: Este estudo visa analisar o efeito agudo do zolpidem em relação às alterações cognitivas e de equilíbrio na população idosa. Métodos: Uma busca em quatro bases de informação científica virtual foi feita por dois pesquisadores independentes e incluiu ensaios clínicos randomizados. Os estudos avaliaram o uso de zolpidem em pacientes idosos. Alterações cognitivas e de equilíbrio foram analisadas. Resultados: Seis artigos foram incluídos. A média de idade entre os estudos foi de 69 anos. As seguintes posologias foram analisadas: 5; 6,25; 10; e 12,5 mg. Em relação às alterações cognitivas, comparando-se zolpidem com placebo, não há diferença estatisticamente significativa entre os grupos. Entretanto, no desfecho alterações de equilíbrio, há diferença estatisticamente significativa entre intervenção e comparação, a favor do placebo. Conclusões: Zolpidem, mesmo em doses usuais (5 e 10 mg), mostrou aumentar o risco para alterações de equilíbrio, entretanto, isso não ocorre em relação às alterações cognitivas.

Palavras-chave: zolpidem, idoso, equilíbrio postural, disfunção cognitiva, revisão sistemática.

\section{INTRODUCTION}

7 olpidem is one of the most internationally

prescribed hypnotic (non-benzodiazepine) agents, a derivative of imidazopyridine, and has a small action as an agonist at GABA-A receptors for treatment. ${ }^{1}$ It is used for sedation, anxiety reduction, and central muscle relaxation, and it has an anticonvulsant effect. ${ }^{2}$ In the elderly population, as they age, the consumption of hypnotics increases. ${ }^{3}$ Between 5 and 33\% of

\footnotetext{
This study was conducted by the Department of Evidence-Based Medicine, Centro Universitário Lusíada, Santos, SP, Brazil.

'Department of Evidence-Based Medicine, Centro Universitário Lusíada - Santos, SP, Brazil.

2Department of Evidence-Based Medicine, Universidade de São Paulo - São Paulo, SP, Brazil.

${ }^{3}$ Department of Surgery, Hospital Israelita Albert Einstein - São Paulo, SP, Brazil.

Guilherme Tavares. Rua Carolino Rodrigues, 17 - Boqueirão - 11055-070 Santos SP - Brazil. E-mail: tavaresguilherme18@gmail.com

Disclosure: The authors report no conflicts of interest.

Funding: none.

Received on January 16, 2021. Accepted in final form on April 10, 2021.
}

\section{(cc) BY}


the ageing population, from North America and the United Kingdom, received a prescription of benzodiazepine or a benzodiazepine receptor agonist for the sleep problem., ${ }^{4,5}$

Regarding balance dysfunctions, the $\mathrm{z}$-drugs (non-benzodiazepine hypnotics) are associated with elderly hospitalization for fractures, injuries, and possibly falls. . $^{6-8}$ Besides that, cognition is critical for functional independence as people age, including whether someone can live independently, manage finances, take medications correctly, and drive safely. In addition, intact cognition is vital for humans to communicate effectively and for social life. ${ }^{9}$ Therefore, balance and cognitive dysfunctions have an important role in the quality of life of the elderly.

Currently, there are still controversies in the scientific literature about cognitive dysfunctions and the increased risk for falls due to zolpidem use in the elderly. ${ }^{8,10,11}$ We performed a systematic review of randomized controlled trials, aiming to analyze the acute effect of zolpidem on cognitive and balance dysfunctions in the elderly population.

\section{METHODS}

This systematic review was submitted in the International Prospective Register of Systematic Reviews (PROSPERO) under trial registry CRD42020196434 and was conducted by the Department of Evidence-Based Medicine, Centro Universitário Lusíada, Santos, SP, Brazil.

The search for evidence was carried out independently by two researchers in the following virtual scientific information bases: Medline (PubMed), EMBASE, Central (Cochrane), Lilacs (VHL), and manual search, evaluating references from primary studies and reviews. The selection of studies was cross-checked and completed on March 4, 2021. The same search strategy was used in all scientific bases: (aged OR elderly OR older adult) AND (zolpidem OR non-benzodiazepine OR non-BZD).

The eligibility criteria were randomized clinical trials analyzing cognitive or balance dysfunctions as primary or secondary outcomes due to the acute effect of zolpidem usage in the elderly population ( $>60$ years); no period or language limit was used; only the available full-text articles were included. The following study data were independently extracted by two researchers: name of the author and year of publication, study design, population studied, methods of intervention and comparison, the absolute number of events, means, deviation, standard error for the exams performed, and follow-up time. Data provided by the studies only in graphics were extracted using the WebPlotDigitizer software. ${ }^{12}$

Randomized clinical trials were assessed for risk of bias according to the following criteria: focal matter, randomization, blindfold allocation, double-blind, losses less than $20 \%$, analysis by intention to treat, prognostic characteristics, outcome (importance, time, and method), sample calculation, and JADAD (scale ranging from 0 to 5 points, which takes into account randomization, double-blinding, and losses).

\section{RESULTS}

As shown in Figure 1, 895 articles were retrieved from Medline (PubMed) database and 2,928 articles from EMBASE, Central (Cochrane), and Lilacs databases. Excluding duplicates (2,148 studies), 1,675 articles remained to be selected; 117 articles were selected by title, 41 articles were selected by abstract, and finally, 6 articles were selected by full text. ${ }^{13-18}$ It was not possible to perform a meta-analysis considering the variety of analyzed outcomes, lack of data, and convergent measures. Qualitative analysis of this systematic review was then performed. The baseline characteristics of the included studies are given in Table 1. The risk of biases is given in Table 2.

Considering all the studies, 145 patients were evaluated, 49 males and 68 females. The mean age of the participants in the studies was approximately 69 years. The mean washout period (crossover studies) between intervention and control (placebo) was approximately 10 days. The following zolpidem dosages were evaluated: $5,6.25,10$, and $12.5 \mathrm{mg}$.

The results were divided into two main outcomes: (1) cognitive dysfunctions and (2) balance dysfunctions, as shown in Tables 3 and 4, respectively.
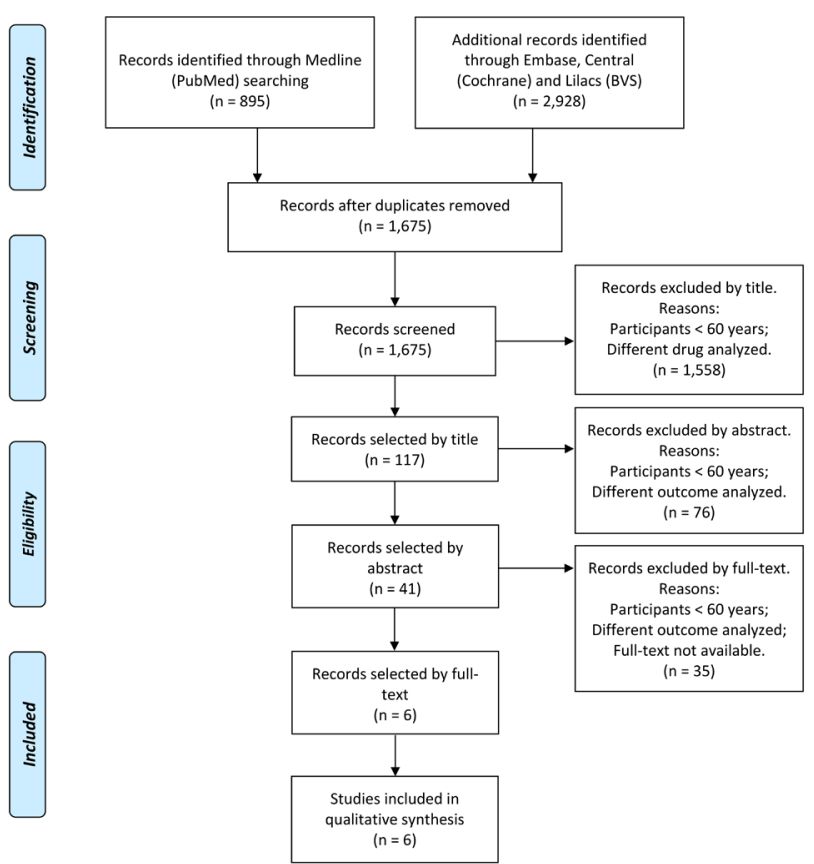

Figure 1. PRISMA flow diagram. 
Table 1. Baseline characteristics of the included studies.

\begin{tabular}{|c|c|c|c|c|c|c|}
\hline $\begin{array}{l}\text { Author and year } \\
\text { of publication }\end{array}$ & Study design & Patients & Intervention & Control & $\begin{array}{l}\text { Outcomes } \\
\text { analyzed }\end{array}$ & Follow-up \\
\hline $\begin{array}{l}\text { Uemura et al., } \\
2015^{13}\end{array}$ & $\begin{array}{l}\text { Randomized } \\
\text { controlled trial } \\
(\mathrm{RCT}) / \text { crossover }\end{array}$ & $\begin{array}{l}\mathrm{n}=13(2 \mathrm{M} \text { and } \\
11 \mathrm{~F}) \text { between } \\
60 \text { and } 70 \\
\text { years, healthy, } \\
\text { time to bed } \\
\text { between } 20 \text { and } \\
24 \mathrm{~h} \text {. }\end{array}$ & $\begin{array}{c}\text { Zolpidem }(5 \mathrm{mg}), \\
\text { Triazolam }(0.125 \mathrm{mg}) \text {, } \\
\text { Rilmazafone }(1 \mathrm{mg}) \\
\text { — administered at } \\
\text { bedtime - with } 6 \text { days } \\
\text { of washout between } \\
\text { treatments }\end{array}$ & $\begin{array}{l}\text { Placebo - } \\
\text { administered } \\
\text { at bedtime - } \\
\text { with } 6 \text { days of } \\
\text { washout between } \\
\text { treatments }\end{array}$ & $\begin{array}{l}\text { CFF, SDR, SMT, } \\
\text { body sway test, } \\
\text { TUG and FRT }\end{array}$ & $\begin{array}{c}\text { Outcomes analysis on } \\
\text { the day after exposure, } \\
6 \text { days of washout - } \\
4 \text { periods }\end{array}$ \\
\hline $\begin{array}{l}\text { Boyle et al., } \\
2009^{14}\end{array}$ & RCT/crossover & $\begin{array}{l}\mathrm{n}=24(7 \mathrm{M} \text { and } \\
17 \mathrm{~F}) \text {, between } \\
65 \text { and } 75 \\
\text { years, healthy, } \\
\text { time to bed } \\
\text { between } 20 \text { and } \\
24 \mathrm{~h} \text {. }\end{array}$ & $\begin{array}{l}\text { Gaboxadol }-10 \mathrm{mg} \\
\text { ( } \mathrm{n}=24) \text { and Zolpidem } \\
\quad-5 \mathrm{mg}(\mathrm{n}=23) \\
\text { - administered at } \\
\text { bedtime }- \text { with } 3 \text { days } \\
\text { of washout between } \\
\text { treatments }\end{array}$ & $\begin{array}{l}\text { Placebo }(\mathrm{n}=23) \\
\text { — administered } \\
\text { at bedtime - } \\
\text { with } 3 \text { days of } \\
\text { washout between } \\
\text { treatments }\end{array}$ & $\begin{array}{l}\text { CFF, body sway } \\
\text { test, and adverse } \\
\text { events }\end{array}$ & $\begin{array}{l}\text { Outcome analysis up } \\
\text { to } 12 \mathrm{~h} \text { post exposure; } \\
3 \text { days of washout } \\
\text { between treatment - } \\
3 \text { periods }\end{array}$ \\
\hline $\begin{array}{l}\text { Zammit et al., } \\
2008^{15}\end{array}$ & RCT/crossover & $\begin{array}{c}\mathrm{n}=11(3 \mathrm{M} \text { and } \\
8 \mathrm{~F}) \text {, between } 65 \\
\text { and } 81 \text { years, } \\
\text { healthy. }\end{array}$ & $\begin{array}{c}\text { Zolpidem }(10 \mathrm{mg})- \\
\mathrm{n}=11\end{array}$ & Placebo $-n=10$ & $\begin{array}{l}\text { SOT and adverse } \\
\text { events }\end{array}$ & $\begin{array}{c}\text { Each treatment lasted } \\
2 \text { days, with a washout } \\
\text { period of } 2 \text { days. }\end{array}$ \\
\hline $\begin{array}{l}\text { Hindmarch et al., } \\
2006^{16}\end{array}$ & RCT/crossover & $\begin{array}{c}\mathrm{n}=24(10 \mathrm{M} \text { and } \\
14 \mathrm{~F}) \text {, between } \\
65 \text { and } 78 \text { years }\end{array}$ & $\begin{array}{l}\text { Zolpidem (6.25 mg) } \\
-\mathrm{n}=23 \text {, Zolpidem } \\
\text { (12.5 mg) }-\mathrm{n}=24 \text { and } \\
\text { Flurazepam }(30 \mathrm{mg})- \\
\mathrm{n}=23\end{array}$ & Placebo $-n=23$ & $\begin{array}{l}\text { CFF, CRT, CTT, } \\
\text { DSST, memory } \\
\text { recall, and adverse } \\
\text { events }\end{array}$ & $\begin{array}{l}\text { Each treatment lasted } \\
2 \text { days, with a washout } \\
\text { period of } 28-42 \text { days. } \\
\text { After } 7-14 \text { days post } \\
\text { exposure, there was a } \\
\text { medical evaluation. }\end{array}$ \\
\hline $\begin{array}{l}\text { Bentué-Ferrer } \\
\text { et al., } 2003^{17}\end{array}$ & RCT/crossover & $\begin{array}{l}\mathrm{n}=49(21 \mathrm{M} \text { and } \\
28 \mathrm{~F}) \text {, between } \\
64 \text { and } 78 \\
\text { years, healthy }\end{array}$ & $\begin{array}{l}\text { Zolpidem }(5 \mathrm{mg}) \text {, } \\
\text { Zopiclone }(3.75 \mathrm{mg}) \text { and } \\
\text { Lormetazepam }(1 \mathrm{mg})\end{array}$ & Placebo & $\begin{array}{l}\text { CTT, LMT, Sternberg } \\
\text { MRT, SRT, body } \\
\text { sway test, and } \\
\text { adverse events }\end{array}$ & $\begin{array}{c}\text { Each treatment lasted } \\
12 \mathrm{~h} \text {, with a washout } \\
\text { period of } 8 \text { days. }\end{array}$ \\
\hline $\begin{array}{l}\text { Fairweather } \\
\text { et al., } 1992^{18}\end{array}$ & RCT/crossover & $\begin{array}{l}n=24(6 \mathrm{M} \text { and } \\
18 \mathrm{~F}) \text {, between } \\
63 \text { and } 80 \\
\text { years, healthy }\end{array}$ & $\begin{array}{l}\text { Zolpidem (5 mg) and } \\
\text { Zolpidem (10 mg) }\end{array}$ & Placebo & $\begin{array}{l}\text { CTT, CRT, Sternberg } \\
\text { MRT, and WRT }\end{array}$ & $\begin{array}{l}\text { Each treatment lasted } \\
7 \text { days ( } 3 \text { treatments in } \\
\text { total), with a washout } \\
\text { period of } 7 \text { days. They } \\
\text { were analyzed after the } \\
\text { first day and the last. }\end{array}$ \\
\hline
\end{tabular}

TUG: The timed up and go; FRT: functional reach test; SDR: simple discrimination reaction; STM: short-term memory; CFF: critical flicker fusion; SOT: sensory organization test; CRT: choice reaction time; CTT: continuous tracking test; DSST: digit symbol substitution test; MRT: mean reaction time; LMT: learning memory task; SRT: simple reaction time; WRT: word recognition task; mg: milligram; M: male; F: female.

Table 2. Bias of included studies.

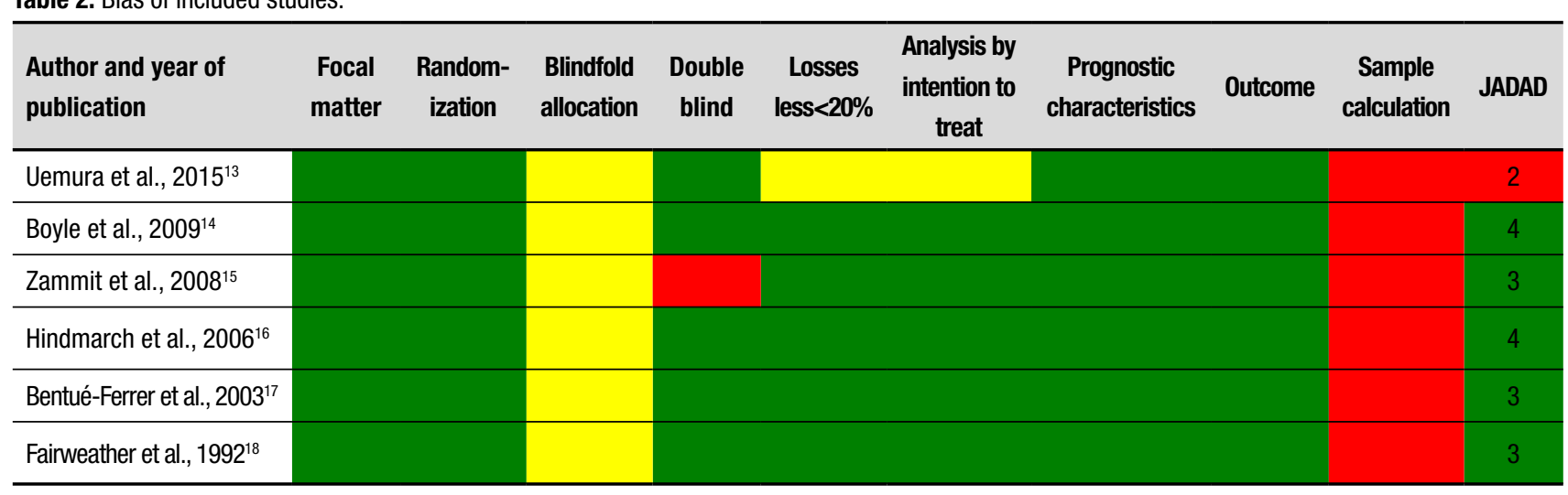

Green shade: absence of bias; red shade: presence of bias; yellow shade: doubt. 
Table 3. Results: cognitive dysfunctions.

\begin{tabular}{|c|c|c|c|c|}
\hline \multirow{2}{*}{$\begin{array}{l}\text { Author and } \\
\text { year of } \\
\text { publication }\end{array}$} & \multirow{2}{*}{$\begin{array}{c}\text { Intervention ' } \\
\text { control }\end{array}$} & \multicolumn{3}{|c|}{ Cognitive dysfunctions } \\
\hline & & CFF (Hz) & CTT & CRT \\
\hline $\begin{array}{l}\text { Uemura et al., } \\
2015^{13}\end{array}$ & $\begin{array}{c}\text { Zolpidem }(5 \mathrm{mg})^{\prime} \\
\text { placebo }\end{array}$ & $\begin{array}{c}\text { I: } 33 \text { (SEM=0.19) } \\
\text { C: } 32.4 \text { (SEM=0.18) }\end{array}$ & No data & No data \\
\hline Boyle et al., $2009^{14}$ & $\begin{array}{c}\text { Zolpidem }(5 \mathrm{mg}) \\
\text { placebo }\end{array}$ & $\begin{array}{c}\text { Zolpidem minus placebo (MD) } \\
1 \mathrm{~h} 30:-0.53 \text { (-1.20 to } 0.14) \\
4 \mathrm{~h}:-0.1(-0.77 \text { to } 0.57) \\
8 \mathrm{~h}:-0.05 \text { (-0.72 to } 0.62)\end{array}$ & No data & No data \\
\hline
\end{tabular}

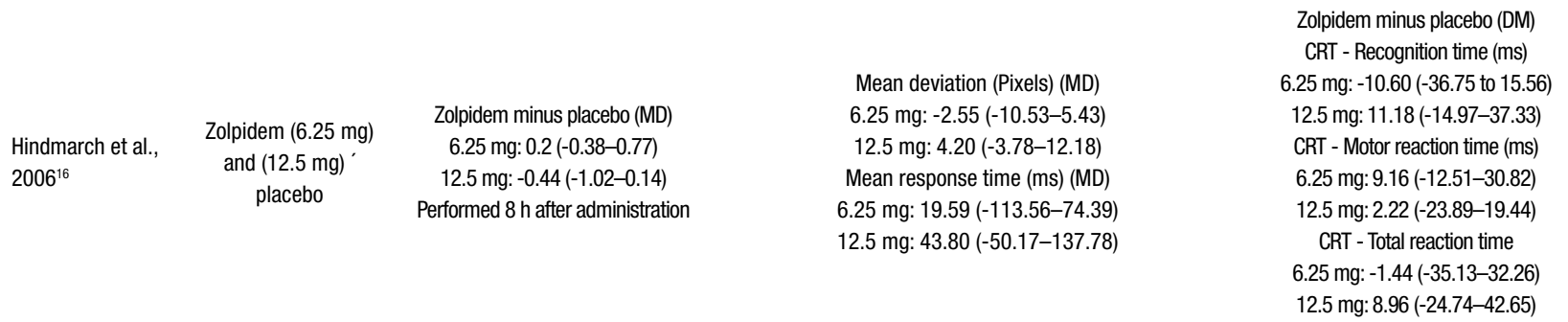

\begin{tabular}{|c|c|c|c|c|}
\hline $\begin{array}{l}\text { Bentué-Ferrer } \\
\text { et al., 2003 }\end{array}$ & $\begin{array}{c}\text { Zolpidem }(5 \mathrm{mg})^{\prime} \\
\text { placebo }\end{array}$ & No data & $\begin{array}{c}\text { Mean }( \pm \text { SEM) }(\mathrm{mm}) \\
\text { HO: I=11.41 }(10.56-12.26) \mathrm{C}=11.92(11.13-12.71) \\
\text { H9: } \mathrm{I}=14.35(12.44-16.26) \\
\mathrm{C}=12.32(11.22-13.42)\end{array}$ & No data \\
\hline
\end{tabular}

Fairweather et al., Zolpidem $(5 \mathrm{mg})$ and $1992^{18}$
$(10 \mathrm{mg})$ ' placebo
No data

\section{Placebo}

RMSTE (units) - Mean (SD): Day 1 - Baseline=11.4 (7.4);

$10 \mathrm{H}=13.3$ (12.4); $12 \mathrm{H}=14.0$ (11.6); $18 \mathrm{H}=13.2$ (11.2); Day

7 - Baseline=13.8 (14.4); 10H=14.0 (16.3); $12 \mathrm{H}=15.2(22.0)$; $18 \mathrm{H}=13.7$ (12.7).

Peripheral stimuli (ms) - Mean (SD): Day 1 - Baseline $=0.43$ $(0.08) ; 10 \mathrm{H}=0.45(0.13) ; 12 \mathrm{H}=0.46(0.08) ; 18 \mathrm{H}=0.46(0.1)$; Day 7 - Baseline $=0.45(0.1) ; 10 \mathrm{H}=0.43(0.08) ; 12 \mathrm{H}=0.44$ $(0.07) ; 18 \mathrm{H}=0.46(0.11)$

Zolpidem (5 mg)

RMSTE (units) - Mean (SD): Day 1 - Baseline=12.7 (10.4); $10 \mathrm{H}=12.8(9.6) ; 12 \mathrm{H}=13.4(9.9) ; 18 \mathrm{H}=13.5$ (10.2); Day

7 - Baseline=11.7 (9.5); 10H=12.6 (9.6); 12H=12.1 (8.5); $18 \mathrm{H}=12.1$ (10.1).

Peripheral stimuli (ms) - Mean (SD): Day $1-$ Baseline $=0.43$ $(0.07) ; 10 \mathrm{H}=0.43(0.1) ; 12 \mathrm{H}=0.46(0.1) ; 18 \mathrm{H}=0.45(0.6) ;$ Day 7 - Baseline $=0.43(0.07) ; 10 \mathrm{H}=0.44(0.07) ; 12 \mathrm{H}=0.45(0.07)$; $18 \mathrm{H}=0.45(0.06)$.

Zolpidem (10 mg)

RMSTE (units) - Mean (SD): Day 1 - Baseline=12.5 (10.8);

$10 \mathrm{H}=13.3(9.9) ; 12 \mathrm{H}=13.2$ (9.9); $18 \mathrm{H}=13.4$ (11.9); Day

7 - Baseline $=11.5(8.1) ; 10 \mathrm{H}=12.4(10.1) ; 12 \mathrm{H}=12.7(11.3)$; $18 \mathrm{H}=13.5$ (12.1).

Peripheral stimuli (ms) - Mean (SD): Day 1 - Baseline $=0.43$ (0.08); $10 \mathrm{H}=0.44(0.07) ; 12 \mathrm{H}=0.46(0.08) ; 18 \mathrm{H}=0.45(0.08)$; Day 7 - Baseline $=0.44(0.08) ; 10 \mathrm{H}=0.44(0.08) ; 12 \mathrm{H}=0.45$ $(0.07) ; 18 \mathrm{H}=0.46(0.09)$.
"There was no statistically significant difference." 
Table 3. Continuation.

\begin{tabular}{|c|c|c|c|c|c|c|c|c|}
\hline \multirow{2}{*}{$\begin{array}{l}\text { Author and } \\
\text { year of } \\
\text { publication }\end{array}$} & \multicolumn{8}{|c|}{ Cognitive dysfunctions } \\
\hline & $\begin{array}{c}\text { Sternberg } \\
\text { MRT }\end{array}$ & SDR & STM & DSST & LMT & SRT & WRT & Memory Recall \\
\hline $\begin{array}{l}\text { Uemura et al., } \\
2015^{13}\end{array}$ & No data & $\begin{array}{c}\text { Accuracy rate (\%) } \\
\text { I: } 94.2 \text { (SEM=1.1) } \\
\text { C: } 92.9 \text { (SEM=0.9) } \\
\text { Reaction time (s) } \\
\text { I: } 0.51(0.01) \\
\text { C: } 0.5(0.01)\end{array}$ & $\begin{array}{l}\text { I: Accuracy rate } \\
(\%)=34.6(2.4) \\
\text { C: Accuracy rate } \\
(\%)=32.1(2.4)\end{array}$ & No data & No data & No data & No data & No data \\
\hline
\end{tabular}

\begin{tabular}{|c|c|c|c|c|c|c|c|}
\hline $\begin{array}{l}\text { Boyle et al., } \\
2009^{14}\end{array}$ & No data & No data & No data & No data & No data & No data & No data \\
\hline
\end{tabular}

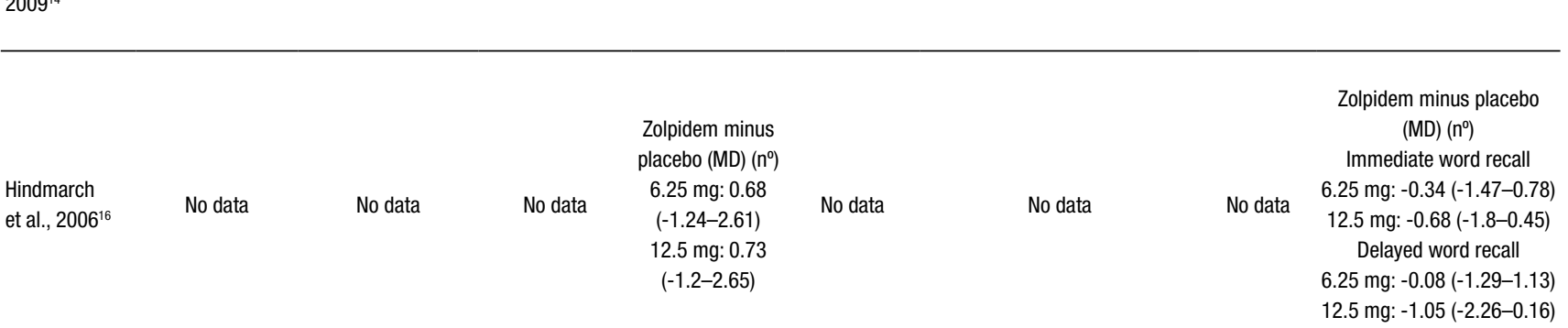

\begin{tabular}{|c|c|c|c|c|c|c|c|c|}
\hline 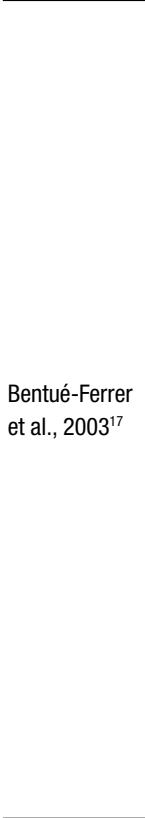 & $\begin{array}{c}\text { Mean (SEM) } \\
2 \text { digits } \\
\text { H0: I=825 (42) } \\
\text { C=985 (62) } \\
\text { H9: I=892 (40) } \\
\text { C=923 (56) } \\
3 \text { digits } \\
\text { H0: I=926 (34) } \\
\text { C=953 (45) } \\
\text { H9: I=933 (33) } \\
\text { C=950 (44) } \\
4 \text { digits } \\
\text { H0: I=982 (39) } \\
\text { C=995 (39) } \\
\text { H9: I=950 (34) } \\
\text { C=997 (41) } \\
5 \text { digits } \\
\text { H0: I=1027 (39) } \\
\text { C=1073 (50) } \\
\text { H9: I=1010(34) } \\
\text { C=1039 (42) } \\
6 \text { digits } \\
\text { H0: I=1123(64) } \\
\text { C=1136 (63) } \\
\text { H9: I=1084 (41) } \\
\text { C=1052 (36) }\end{array}$ & No data & No data & No data & 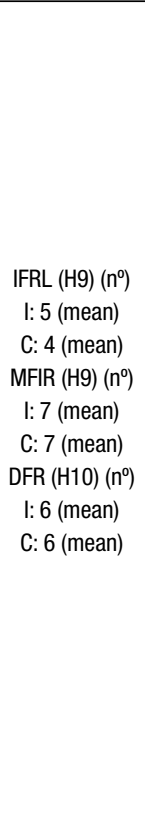 & 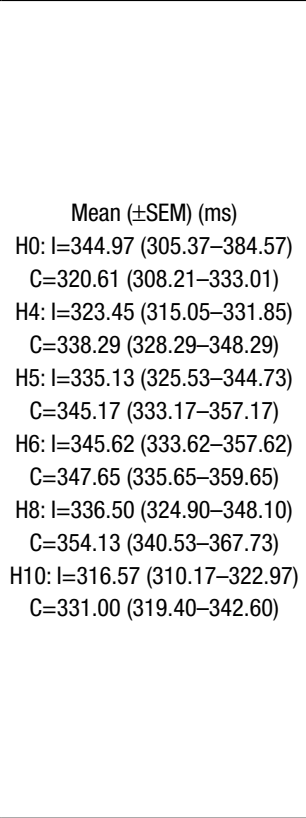 & No data & No data \\
\hline
\end{tabular}

\begin{tabular}{|c|c|c|c|c|c|c|c|c|}
\hline $\begin{array}{l}\text { Fairweather } \\
\text { et al., } 1992^{18}\end{array}$ & $\begin{array}{c}\text { "There was } \\
\text { no statistically } \\
\text { significant } \\
\text { difference." }\end{array}$ & No data & No data & No data & No data & No data & $\begin{array}{c}\text { "There } \\
\text { was no } \\
\text { statistically } \\
\text { significant } \\
\text { difference." }\end{array}$ & No data \\
\hline
\end{tabular}

SDR test: simple discrimination reaction; STM test: short-term memory; CFF: critical flicker fusion; CRT: choice reaction time; CTT: continuous tracking test; DSST: digit symbol substitution test; MRT: mean reaction time; LMT: learning memory task; SRT: simple reaction time; WRT: word recognition task; SD: standard deviation; MD: mean difference; mS: meter per second; RMSTE: root mean square tracking

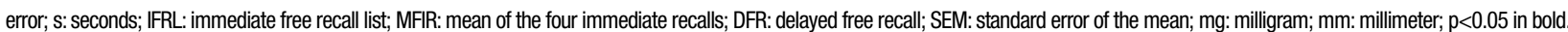


Table 4. Results: Balance dysfunctions.

\begin{tabular}{|c|c|c|c|c|c|}
\hline \multirow{2}{*}{$\begin{array}{l}\text { Author and year } \\
\text { of publication }\end{array}$} & \multirow{2}{*}{$\begin{array}{c}\text { Intervention ' } \\
\text { control }\end{array}$} & \multicolumn{4}{|c|}{ Balance dysfunctions } \\
\hline & & Body sway test & TUG & FRT & NeuroCom EquiTest SOT \\
\hline $\begin{array}{l}\text { Uemura et al., } \\
2015^{13}\end{array}$ & $\begin{array}{l}\text { Zolpidem }(5 \mathrm{mg})^{\prime} \\
\quad \text { placebo }\end{array}$ & $\begin{array}{c}\text { Mean (SEM) }(\mathrm{cm}) \\
\text { Eyes open } \\
\text { I: } 141.7(1.7) \\
\text { C: } 143.4(1.7) \\
\text { Eyes closed } \\
\text { I: } 171.9(2.3) \\
\text { C: } 172.3(2.3) \\
\end{array}$ & $\begin{array}{c}\text { Mean (SEM) } \\
(\mathrm{s}) \\
\mathrm{I}: 7.61 \\
(0.05) \\
\text { C: } 7.67 \\
(0.05)\end{array}$ & $\begin{array}{c}\text { Mean (SEM) } \\
(\mathrm{cm}) \\
\text { I: } 311.8 \\
(4.0) \\
\text { C: } 288.4 \\
(4.2)\end{array}$ & No data \\
\hline Boyle et al., $2009^{14}$ & $\begin{array}{l}\text { Zolpidem }(5 \mathrm{mg})^{\prime} \\
\quad \text { placebo }\end{array}$ & $\begin{array}{c}\text { Zolpidem minus placebo (GM) }\left(\mathrm{cm}^{2}\right. \\
\text { Eyes open } \\
1 \mathrm{~h} 30: 2.00(1.62-2.46) \\
4 \mathrm{~h}: 1.78(1.44-2.20) \\
8 \mathrm{~h}: 1.23(1.00-1.52) \\
\text { Eyes closed } \\
1 \mathrm{~h} 30: 1.65(1.33-2.04) \\
4 \mathrm{~h}: 1.38(1.11-1.71) \\
8 \mathrm{~h}: 1.09(0.88-1.35) \\
\end{array}$ & No data & No data & No data \\
\hline $\begin{array}{l}\text { Zammit et al., } \\
2008^{15}\end{array}$ & $\begin{array}{l}\text { Zolpidem }(10 \mathrm{mg})^{\prime} \\
\text { placebo }\end{array}$ & No data & No data & No data & $\begin{array}{c}\text { LS mean (SE) }(\%) \\
\text { ES1: }-6.67 \pm 2.02 \\
\text { ES2: }-7.41 \pm 1.90 \\
\text { ES3: }-6.35 \pm 2.78 \\
\text { ES4: }-13.14 \pm 3.76 \\
\text { ES5: }-27.69 \pm 5.38 \\
\text { ES6: }-17.21 \pm 3.61 \\
\text { CES: }-15.83 \pm 2.88 \\
\text { Somatosensory ratio: }-1.74 \pm 1.60 \\
\text { Visual ratio: }-8.76 \pm 4.00 \\
\text { Vestibular ratio: }-27.43 \pm 5.91 \\
\text { Preference ratio: } 12.82 \pm 5.90 \\
\end{array}$ \\
\hline $\begin{array}{l}\text { Bentué-Ferrer } \\
\text { et al., } 2003^{17}\end{array}$ & $\begin{array}{l}\text { Zolpidem }(5 \mathrm{mg})^{\prime} \\
\quad \text { placebo }\end{array}$ & 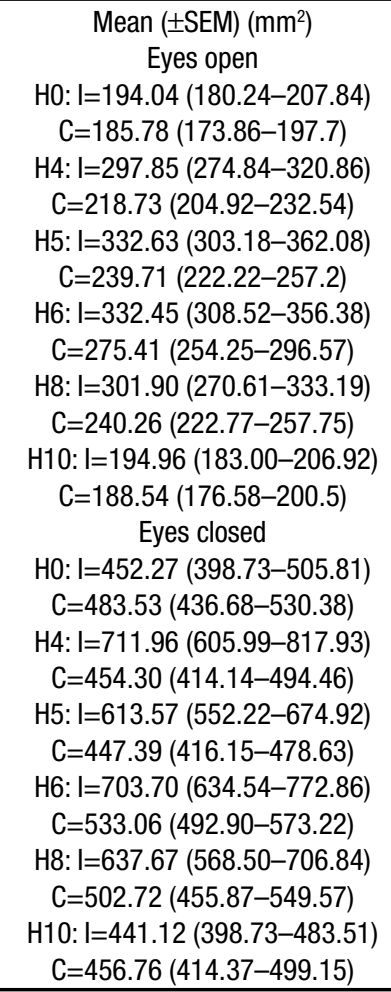 & No data & No data & No data \\
\hline
\end{tabular}

TUG: the timed up and go; FRT: functional reach test; ES: equilibrium score; CES: composite equilibrium score; SOT: sensory organization test; SEM: standard error of the mean; GM: geometric mean; cm: centimeter; mg: milligram; s: seconds; LS: least square; SE: standard error; $\mathrm{p}<0.05$ in bold. 


\section{Cognitive dysfunctions}

\section{Memory}

\section{Short-term memory}

Four studies ${ }^{13,16-18}$ analyzed this outcome by carrying out five tests (see Table 3): Sternberg mean reaction time (MRT), word recognition task (WRT), short-term memory (STM), memory recall, and learning memory task (LMT).

The obtained data showed no statistically significant difference between zolpidem $(5,6.25,10$, and $12.5 \mathrm{mg})$ and placebo in relation to short-term memory.

\section{Long-term memory}

Two studies ${ }^{16,17}$ assessed this outcome by carrying out two tests (see Table 3): Memory recall and learning memory task (LMT).

The obtained results demonstrated no statistically significant difference between zolpidem $(5,6.25$, and $12.5 \mathrm{mg}$ ) and placebo in relation to long-term memory.

\section{Psychomotricity}

Four studies ${ }^{13,16-18}$ analyzed this outcome by carrying out three tests (see Table 3): Continuous tracking test (CTT), choice reaction time (CRT), and simple discrimination reaction (SDR).

The obtained data demonstrated no statistically significant difference between zolpidem $(5,6.25,10$, and $12.5 \mathrm{mg}$ ) and placebo in relation to psychomotricity.

\section{Vision}

Four studies ${ }^{13,14,16,17}$ analyzed this outcome by carrying out two tests (see Table 3): Critical flicker fusion (CFF) and simple reaction time (SRT).

Uemura et al. ${ }^{13}$ performed the CFF and showed a statistically significant difference between zolpidem $(5 \mathrm{mg})$ and placebo, in favor of zolpidem. Three studies ${ }^{14,16,17}$ demonstrated no statistically significant difference between zolpidem $(5,6.25$, and $12.5 \mathrm{mg})$ and placebo in relation to vision changes.

\section{Processing, attention, and concentration}

One study ${ }^{16}$ assessed this outcome by carrying out the digit symbol substitution test (DSST) (see Table 3).

The obtained results showed no statistically significant difference between zolpidem ( 6.25 and $12.5 \mathrm{mg}$ ) and placebo in relation to processing, attention, and concentration.

\section{Balance dysfunctions}

Four studies ${ }^{13-15,17}$ analyzed this outcome by carrying out four tests (see Table 4): Body sway test, the timed up and go (TUG), functional reach test (FRT), and NeuroCom EquiTest sensory organization test (SOT).

Uemura et al. ${ }^{13}$ performed two tests (body sway test and TUG) and showed no statistically significant difference between zolpidem $(5 \mathrm{mg})$ and placebo in relation to balance dysfunctions. Regarding the FRT, the same study demonstrated a statistically significant difference between zolpidem ( $5 \mathrm{mg}$ ) and placebo, in favor of zolpidem.

Two studies ${ }^{14,17}$ carried out the body sway test and showed a statistically significant difference between zolpidem (5 $\mathrm{mg}$ ) and placebo, in favor of placebo with eyes open and closed at $1 \mathrm{~h}$ and $30 \mathrm{~min}, 4 \mathrm{~h}, 5 \mathrm{~h}, 6 \mathrm{~h}$, and $8 \mathrm{~h}$ after the zolpidem, and placebo administration.

Zammit et al. ${ }^{15}$ performed the NeuroCom EquiTest SOT and showed a statistically significant difference between zolpidem $(10 \mathrm{mg})$ and placebo, in favor of placebo in conditions $1,2,4,5$, and 6 , as well as in the balance score (CES) and in the vestibular rate.

\section{DISCUSSION}

This study is a systematic review of randomized controlled trials assessing the acute impact of zolpidem on cognitive and balance dysfunctions in the elderly population. This study showed that the use of zolpidem in the elderly is not related to cognitive dysfunctions. However, zolpidem, even in the usual dosages ( 5 and 10 $\mathrm{mg}$ ) may lead to balance dysfunctions.

The use of zolpidem in the elderly with sleep disorders provides better sleep quality, adding an independent and significant contribution to the quality of life beyond psychopathological symptoms. ${ }^{19}$ Besides that, the effects of sleep disorders are associated with a wide range of health conditions, including an increased risk of high blood pressure, diabetes, obesity, major depression, heart attack, and stroke. ${ }^{20}$ Thus, the aim of this study is to guarantee the quality of sleep and avoid such diseases without impairing cognition, whose integrity is of utmost importance to maintain communicative skills and, therefore, social life. ${ }^{9}$

This study showed no evidence in the scientific literature supporting any cognitive dysfunctions due to the use of zolpidem in the elderly population. However, recent randomized controlled trials evidenced a cognitive impairment relating to zolpidem in non-elderly. ${ }^{21-23}$ Stranks et al. performed a systematic review and showed that the performance on attention, verbal memory, and psychomotor speed were impaired compared to the control group, based on the data of middle-aged participants (mean age was 37 years) who ingested zolpidem before bedtime. Other cognition domains, such as speed of processing and working memory, were not affected. ${ }^{24}$ 
In addition to that, physicians should be aware of the greater susceptibility of the elderly to the negative consequences of zolpidem use and of other sedative hypnotics, especially the potential risk for falls, due to lower clearance rates and higher maximum serum concentration of these drugs. ${ }^{25}$ Fractures and injuries impair the functional capacity and the health-related quality of life in the long-term follow-up. ${ }^{8,26}$ Consequently, physicians should limit the treatment of these drugs when possible or use the lowest dose possible for patients at higher risk of imbalance. Chronic motor dysfunction of stroke patients ${ }^{27}$ and diabetic peripheral neuropathic patients ${ }^{28}$ present high individual risk of falling, and consequently, zolpidem should be used with caution in these patients. Also, osteoporotic patients are associated with an increased risk of related injuries, ${ }^{29,30}$ and zolpidem use should also be taken with precaution.

High-risk group patients should receive differentiated care, especially in activities at home, avoiding possible falls and encouraging certain physical exercises. de Kam et al. ${ }^{31}$ showed that exercise has positive effects on important predictors for falls and fractures, such as muscle strength, balance, and bone mineral density.

This study has some limitations. The included studies present a small number of patients, different doses of zolpidem, different treatment periods, and different analysis dates. Also, the studies used heterogeneous tests for the estimation of balance and cognition performances, which is why it was not possible to perform the meta-analysis.

The future of clinical research on this topic should stick to new randomized clinical trials, standardizing the tests for cognition and balance performed on the elderly population with a bigger sample size, allowing, then, a quantitative analysis of future systematic reviews. Future studies will allow us to consider the use of medication, by calculating the number needed to treat (NNT) or harm (NNH) to find out if the effectiveness in controlling insomnia and quality of life really outweighs the risks of cognitive and balance disorders.

The results of this study demonstrate that there is no statistically significant difference between zolpidem (5, $6.25,10$, and $12.5 \mathrm{mg}$ ) and placebo regarding cognitive dysfunctions (memory, psychomotricity, processing, attention, concentration, and vision). However, related to balance dysfunctions, the comparison between zolpidem (5 and $10 \mathrm{mg}$ ) and placebo showed that most articles present a statistically significant difference in favor of placebo, demonstrating that this drug produces relevant balance dysfunctions.

Authors' contributions. GT: methodology, data curation, software, validation, formal analysis, investigation, writing (original draft), writing (review and editing), visualization, and project administration. GK: conceptualization, methodology, supervision, and project administration. FT: validation, data curation, formal analysis, investigation, writing (original draft), writing (review and editing), visualization, supervision, and project administration. CNT: methodology, writing (original draft), and writing (review and editing). BRBS: methodology and writing (original draft). BMACB, DBW, ECB, GSV, GB, GRCL, and JGAF: methodology. WMB: project administration.

\section{REFERENCES}

1. Sanna E, Busonero F, Talani G, Carta M, Massa F, Peis M, et al. Comparison of the effects of zaleplon, zolpidem, and triazolam at various GABAA receptor subtypes. Eur J Pharmacol. 2002;451(2):103-10. https://doi. org/10.1016/S0014-2999(02)02191-X

2. Salvà $P$, Costa J. Clinical pharmacokinetics and pharmacodynamics of zolpidem. Clin Pharmacokinet. 1995;29(3):142-53. https://doi. org/10.2165/00003088-199529030-00002

3. Wortelboer U, Cohrs S, Rodenbeck A, Rüther E. Tolerability of hypnosedatives in older patients. Drugs Aging. 2002;19(7):529-39. https://doi. org/10.2165/00002512-200219070-00006

4. Aparasu R, Mort J, Brandt H. Psychotropic prescription use by communitydwelling elderly in the United States. J Am Geriatr Soc. 2003;51(5):671-7. https://doi.org/10.1034/j.1600-0579.2003.00212.x

5. Craig D, Passmore A, Fullerton K, Beringer T, Gilmore D, Crawford V, et al. Factors influencing prescription of CNS medications in different elderly populations. Pharmacoepidemiol Drug Saf. 2003;12(5):383-7. https:// doi.org/10.1002/pds.865

6. Yu N, Chen P, Tsai H, Huang C, Chiu Y, Tsay W, et al. Association of benzodiazepine and Z-drug use with the risk of hospitalisation for fall-related injuries among older people: a nationwide nested case-control study in Taiwan. BMC Geriatr. 2017;17(1):140. https://doi.org/10.1186/s12877-017-0530-4

7. Berry S, Lee Y, Cai S, Dore D. Nonbenzodiazepine sleep medication use and hip fractures in nursing home residents. JAMA Int Med. 2013;173(9):754. https://doi.org/10.1001/jamainternmed.2013.3795
8. Treves N, Perlman A, Kolenberg Geron L, Asaly A, Matok I. Z-drugs and risk for falls and fractures in older adults - a systematic review and meta-analysis. Age Ageing. 2017;47(2):201-8. https://doi.org/10.1093/ ageing/afx167

9. Murman D. The impact of age on cognition. Semin Hear. 2015;36(03):11121. https://doi.org/10.1055/s-0035-1555115

10. Machado F, Louzada L, Cross N, Camargos E, Dang-Vu T, Nóbrega O. More than a quarter century of the most prescribed sleeping pill: Systematic review of zolpidem use by older adults. Exp Gerontol. 2020;136:110962. https://doi.org/10.1016/j.exger.2020.110962

11. Kaufmann C, Moore A, Bondi M, Murphy J, Malhotra A, Hart L. Association between the use of non-benzodiazepine hypnotics and cognitive outcomes: a systematic review. Curr Sleep Med Rep. 2020;6(1):11-20. https://doi.org/10.1007/s40675-020-00163-1

12. WebPlotDigitizer - Extract data from plots, images, and maps. Automeris. io. 2020 [cited Jun 28, 2020]. Available from: https://automeris.io/WebPlotDigitizer

13. Uemura S, Kanbayashi T, Wakasa M, Satake M, Ito W, Shimizu K, et al. Residual effects of zolpidem, triazolam, rilmazafone and placebo in healthy elderly subjects: a randomized double-blind study. Sleep Med. 2015;16(11):1395-402. https://doi.org/10.1016/j.sleep.2015.05.021

14. Boyle J, Danjou P, Alexander R, Calder N, Gargano C, Agrawal N, et al. Tolerability, pharmacokinetics and night-time effects on postural sway and critical flicker fusion of gaboxadol and zolpidem in elderly subjects. 
Br J Clin Pharmacol. 2009;67(2):180-90. https://doi.org/10.1111/j. 1365-2125.2008.03331.x

15. Zammit G, Wang-Weigand S, Peng X. Use of computerized dynamic posturography to assess balance in older adults after nighttime awakenings using zolpidem as a reference. BMC Geriatr. 2008;8(1):15. https://doi. org/10.1186/1471-2318-8-15

16. Hindmarch I, Legangneux E, Stanley N, Emegbo S, Dawson J. A double-blind, placebo-controlled investigation of the residual psychomotor and cognitive effects of zolpidem-MR in healthy elderly volunteers. Br J Cli Pharmacol. 2006;62(5):538-45. https://doi.org/10.1111/j. 1365-2125.2006.02705.x

17. Bentué-Ferrer D, Allain H, Tarral A, Gandon J. Effects on postural oscillation and memory functions of a single dose of zolpidem $5 \mathrm{mg}$, zopiclone $3.75 \mathrm{mg}$ and lormetazepam $1 \mathrm{mg}$ in elderly healthy subjects. A randomized, cross-over, double-blind study versus placebo. Eur J Clin Pharmacol. 2003;59(3):179-88. https://doi.org/10.1007/ s00228-003-0591-5

18. Fairweather D, Kerr J, Hindmarch I. The effects of acute and repeated doses of zolpidem on subjective sleep, psychomotor performance and cognitive function in elderly volunteers. Eur J Clin Pharmacol. 1992;43(6):597-601. https://doi.org/10.1007/bf02284957

19. Marques D, Meia-Via A, da Silva C, Gomes A. Associations between sleep quality and domains of quality of life in a non-clinical sample: results from higher education students. Sleep Health. 2017;3(5):348-56. https://doi. org/10.1016/j.sleh.2017.07.004

20. Institute of Medicine. Sleep disorders and sleep deprivation: an unmet public health problem. Washington, DC: Institute of Medicine; 2006. https://doi.org/10.17226/11617

21. Matsunaga $Y$, Tagaya H, Fukase $Y$, Hakamata $Y$, Murayama N, Kumaga $\mathrm{Y}$, et al. Effects of zolpidem/triazolam on cognitive performance 12 hours after acute administration. Sleep Med. 2018:52:213-8. https://doi.org/10.1016/j.sleep.2018.06.011

22. Dinges D, Basner M, Ecker A, Baskin P, Johnston S. Effects of zolpidem and zaleplon on cognitive performance after emergent morning awakenings at Tmax: a randomized placebo-controlled trial. Sleep. 2018;42(3);zsy258. https://doi.org/10.1093/sleep/zsy258

23. Neylan T, Richards A, Metzler T, Ruoff L, Varbel J, O'Donovan A, et al. Acute cognitive effects of the hypocretin receptor antagonist almorexant relative to zolpidem and placebo: a randomized clinical trial. Sleep. 2020;43(10):zsaa080. https://doi.org/10.1093/sleep/zsaa080

24. Stranks E, Crowe S. The acute cognitive effects of zopiclone, zolpidem, zaleplon, and eszopiclone: a systematic review and meta-analysis. J Clin Exp Neuropsychol. 2014;36(7):691-700. https://doi.org/10.1080/13803 395.2014 .928268

25. Glass J, Lanctôt K, Herrmann N, Sproule B, Busto U. Sedative hypnotics in older people with insomnia: meta-analysis of risks and benefits. BMJ. 2005;331(7526):1169. https://doi.org/10.1136/bmj.38623.768588.47

26. Willig R. Mortality and quality of life after trochanteric hip fracture. Public Health. 2001;115(5):323-7. https://doi.org/10.1016/s0033-3506(01)00470-x

27. Jørgensen $\mathrm{H}$, Nakayama $\mathrm{H}$, Raaschou $\mathrm{H}$, Olsen T. Recovery of walking function in stroke patients: The Copenhagen stroke study. Arch Phys Med Rehabil. 1995;76(1):27-32. https://doi.org/10.1016/s00039993(95)80038-7

28. Schwartz A, Vittinghoff E, Sellmeyer D, Feingold K, Rekeneire N, Strotmeyer $\mathrm{E}$ et al. Diabetes-related complications, glycemic control, and falls in older adults. Diabetes Care. 2007;31(3):391-6. https://doi.org/10.2337/ dc07-1152

29. Cheong H, Peh W, Guglielmi G. Imaging of diseases of the axial and peripheral skeleton. Radiol Clin North Am. 2008;46(4):703-33. https:// doi.org/10.1016/j.rcl.2008.04.007

30. Guglielmi G, Muscarella S, Leone A, Peh W. Imaging of metabolic bone diseases. Radiol Clin North Am. 2008;46(4):735-54. https://doi. org/10.1016/.rcl.2008.04.010

31. de Kam D, Smulders E, Weerdesteyn V, Smits-Engelsman B. Exercise interventions to reduce fall-related fractures and their risk factors in individuals with low bone density: a systematic review of randomized controlled trials. Osteoporos Int. 2009;20(12):2111-25. https://doi.org/10.1007/ s00198-009-0938-6 